\title{
Pacific
}

Journal of

Mathematics

\section{A NON-HAKEN HYPERBOLIC 3-MANIFOLD COVERED BY A SURFACE BUNDLE}

ALAN W. REID 


\title{
A NON-HAKEN HYPERBOLIC 3-MANIFOLD COVERED BY A SURFACE BUNDLE
}

\author{
ALAN W. REID
}

\begin{abstract}
The question as to whether a finite volume hyperbolic 3-manifold has a finite cover which fibers over the circle, seems, at present, completely mysterious. In this paper we give the first explicit examples of non-Haken hyperbolic 3-manifolds covered by a manifold that fibers of the circle. The methods used are arithmetic using the theory of quaternion algebras.
\end{abstract}

1. Introduction. One of the outstanding unsolved questions in the theory of hyperbolic 3-manifolds is whether every closed hyperbolic 3-manifold has finite cover with positive first betti number. A much stronger question of Thurston (see [28, Question 18]), which if answered affirmatively would imply an affirmative solution to the previous question is whether every finite volume hyperbolic 3-manifold has finite cover which fibers over the circle, the fiber being a compact surface possibly with punctures. There is significant evidence in the first case to support that the conjectured answer to the first question is "yes". However the second situation still seems, at present, completely mysterious.

There is a simple way to construct (closed) Haken hyperbolic 3-manifolds which do not fiber over the circle, but have a double cover which does. Namely one can form the union of two twisted I-bundles over a non-orientable surface (see [10, Chapter 10] for definitions). By unwrapping the I-bundle in a double cover, one obtains a manifold which fibers over the circle, see [10, Chapter 11]. It is not hard to control that the monodromy in the double cover be pseudo-Anosov, and hence the manifolds are hyperbolic by [29]. (See Theorem 2 for some specific examples.) 
Of more interest, is an example due to Gabai [8], of a hyperbolic link complement in $S^{3}$ which does not fiber over the circle, is double covered by a manifold that does, but does not arise from the elementary construction just described. The aim of this paper is to give an explicit example of a non-Haken hyperbolic 3-manifold which has a finite cover which fibers over the circle. To our knowledge no such explicit example seems known and it seems worthwhile to record an example for future study. We remark that for Seifert fibered manifolds the question of whether a manifold can be covered by a manifold that fibers over the circle can be answered in terms of the rational Euler number of the Seifert fibration and the orbifold Euler characteristic of the base, cf. [8] for instance.

Our construction although elementary, uses the theory of arithmetic hyperbolic 3-manifolds for which some explanation will be required. Indeed, we give a pair of commensurable non-Haken hyperbolic 3-manifolds which are covered by a manifold that fibers over the circle. One is the 4-fold cyclic branched cover of the figure eight knot and the other is a manifold that has attracted previous interest as it has volume equal to that of the regular ideal tetrahedron in $\mathbf{H}^{3}$. We shall show that both these manifolds have 10 -fold covers which fiber over the circle, with the fiber having genus 2 . The method would also seem to yield other examples.

\section{Preliminaries on arithmetic Kleinian groups.}

2.1. Arithmetic Kleinian groups are obtained as follows (cf. [5] and [31, Chapter 4]; see [31] for details on quaternion algebras).

Let $k$ be a number field with one complex place and $A$ a quaternion algebra over $k$ ramified at all real embeddings (this means $A \otimes_{k} \mathbf{R}$ is the algebra of Hamiltonian quaternions for each real embedding of $k \hookrightarrow \mathbf{R}$ ). Let $\mathcal{O}$ be an order of $A$ and $\mathcal{O}^{1}$ the group of elements of norm 1 in $\mathcal{O}$. The complex place $k \hookrightarrow \mathbf{C}$ induces an embedding $\rho: A \hookrightarrow M(2, \mathbf{C})$ which restricts to $\rho: \mathcal{O}^{1} \hookrightarrow S L(2, \mathbf{C})$. Then $P \rho\left(\mathcal{O}^{1}\right)$ is a Kleinian group of finite covolume. An arithmetic Kleinian group is one commensurable with a group of the type $P \rho\left(\mathcal{O}^{1}\right)$. We say it is derived from a quaternion algebra if it is actually a subgroup of some $P \rho\left(\mathcal{O}^{1}\right)$. We call $M=\mathbf{H}^{3} / \Gamma$ arithmetic or derived from a quaternion algebra if $\Gamma$ is arithmetic or derived from a quaternion algebra. 
Notation. We will adopt the notation of [31] for quaternion algebras. Briefly, if $K$ is a field of characteristic different from 2, then the symbol $\left(\frac{a, b}{K}\right)$, denotes the quaternion algebra over $K$ with standard basis $\{1, i, j, k\}$ with $i^{2}=a, j^{2}=b$ and $i j=-j i$ where $a$ and $b$ are non-zero elements of $K$.

2.2. We shall make use of of the characterization theorems for arithmetic Kleinian groups given in [19] and [22]. Before stating these, recall some terminology defined in $[\mathbf{2 0}]$ and $[\mathbf{2 1}]$.

Let $\Gamma$ be a non-elementary Kleinian group and $\Gamma^{(2)}=g p\left\{\gamma^{2}: \gamma \in\right.$ $\Gamma\}$. The invariant trace-field of $\Gamma$ is the field $\mathbf{Q}\left(\operatorname{tr} \Gamma^{(2)}\right)$, and denoted by $k \Gamma$. The invariant quaternion algebra of $\Gamma$ is the quaternion algebra defined over $k \Gamma$ given by $\left\{\sum a_{i} \gamma_{i}: a_{i} \in k \Gamma, \gamma_{i} \in \Gamma^{(2)}\right\}$ where all sums are finite. That this is a quaternion algebra is proved in [3]. We denote this algebra by $A \Gamma$. The pair $(A \Gamma, k \Gamma)$ is an invariant of the commensurablity class of $\Gamma$, cf. $[20]$ and $[21]$.

TheOREM A. Let $\Gamma$ be a Kleinian group of finite covolume. Then $\Gamma$ is arithmetic if and only if $\Gamma^{(2)}$ is derived from a quaternion algebra.

ThEOREM B. With $\Gamma$ as in Theorem $A$. $\Gamma$ is derived from a quaternion algebra if and only if the following conditions hold:

(i) $k \Gamma$ has one complex place;

(ii) the set $\operatorname{tr} \Gamma$ consists of algebraic integers;

(iii) $A \Gamma$ is ramified at every real embedding of $k \Gamma$.

Remarks. (1.) If condition (ii) of Theorem B is satisfied we say $\Gamma$ has integral traces.

(2.) The verification of (iii) of Theorem B is simplified by the fact that if $\gamma$ and $\delta$ are a pair of non-commuting loxodromic elements in $\Gamma$ then $\left\{1, \gamma^{2}, \delta^{2}, \gamma^{2} \delta^{2}\right\}$ is a $k \Gamma$ basis for $A \Gamma$. Using this it is easily shown, cf. [14] or [26] that $A \Gamma$ is isomorphic to the quaternion algebra $\left(\frac{a, b}{k \Gamma}\right)$, where $a=\operatorname{tr}(\gamma)^{2}\left(\operatorname{tr}(\gamma)^{2}-4\right)$ and $b=\left(\operatorname{tr}\left(\gamma^{2}\right)+\right.$ $2)\left(\operatorname{tr}\left(\delta^{2}\right)+2\right)(\operatorname{tr}([\gamma, \delta])-2)$. 


\section{2-Orbifold bundles over the circle.}

3.1. Our construction of a manifold $M=\mathbf{H}^{3} / \Gamma$ that has a finite cover which is fibered over the circle proceeds by constructing an orbifold $Q=\mathbf{H}^{3} / \Gamma_{Q}$ which is commensurable with $M$ and such that $\Gamma_{Q}$ contains a normal subgroup $F$ isomorphic to a Fuchsian group of signature $(1 ; 2)$. In particular, $F$ will be geometrically infinite. Topologically $Q$ contains an embedded non-separating incompressible sub-2-orbifold, and $Q$ should be viewed as a 2-orbifold bundle over the circle with fiber a torus with a single cone point of cone angle $\pi$. Thus if $M_{1}$ is the finite cover of $M$ given by $\mathbf{H}^{3} / \Gamma \cap \Gamma_{Q}$ then $M_{1}$ will fiber over the circle as desired.

In view of the previous paragraph, let us make the following definition:

Definition. Let $Q$ be a finite volume hyperbolic 3-orbifold that contains an embedded non-separating incompressible sub-2-orbifold $S$, such that $\pi_{1}^{\text {orb }}(S)$ is a normal subgroup of $\pi_{1}^{\text {orb }}(Q)$. In this case we shall call $Q$ a hyperbolic 2-orbifold bundle over the circle or simply a 2-orbifold bundle. We shall also refer to $S$ as a fiber.

The notion of a 2-orbifold bundle over the circle is completely consistent with that of surface bundles over the circle. For the orbifold fundamental group of every hyperbolic 2-orbifold bundle contains a torsion free subgroup of finite index, and by Stalling's fibration theorem, [25], the quotient of $\mathbf{H}^{3}$ by such a group will be a surface bundle over the circle.

3.2. In this sub-section we collect together some observations about hyperbolic 2-orbifold bundles following in the spirit of Thurston and Jørgenson ([29], [16] and [17]).

Let $M$ be a cusped hyperbolic 3-manifold that fibers over the circle. That is; $M$ is the mapping torus of a pseudo-Anosov selfhomeomorphism $\phi$ of a connected orientable surface with at least one boundary component, say $S$ (see [29]). For notational convenience, in what follows we shall stick to the case where $S$ has a single boundary component, so that the genus $g$ of $S$ is at least 1 . The fundamental group of $\partial M$ is generated by the boundary of a fiber denoted $\ell$ and a section to the fibering induced on $\partial M$, denoted by $t . \quad \ell$ is determined up to orientation, but a choice for $t$ 
has to be made. In any event, on choosing a standard set of generators $a_{1}, b_{1}, \ldots, a_{g}, b_{g}$ for $S$ so that $\ell$ is given by $\prod_{i=1}^{g}\left[a_{i}, b_{i}\right]$, the fundamental group of $M$ can be written as an HNN-extension:

$$
\left\langle t, a_{i}, b_{i} \mid t a_{i} t^{-1}=\phi_{*}\left(a_{i}\right), t b t^{-1}=\phi_{*}\left(b_{i}\right), 1 \leq i \leq g\right\rangle .
$$

With this notation we have.

LEMMA 1. Let $M$ be as above. Then for large enough positive integers $q$ the result of $(0, q)$-Dehn filling on $M$, with respect to the framing described above, produces a hyperbolic 3-orbifold which is a 2-orbifold bundle over the circle, with fiber a surface of genus $g$ with a single cone point of cone angle $2 \pi / q$.

Proof. Let $\phi$ be the monodromy of $M$. Performing $(0, q)$-Dehn filling for large enough positive integers $q$ produces a hyperbolic 3-orbifold by Thurston's Hyperbolic Dehn Surgery Theorem, [27]. The effect of this Dehn filling on the fiber is simply to compactify the punctured surface to a surface of genus $g$ with a single cone point of cone angle $2 \pi / q$. The 2 -orbifold is clearly embedded, nonseparating and incompressible. At the group level we have added the relation $l^{q}=1$. In effect we have therefore simply obtained a cocompact Kleinian group with a normal subgroup isomorphic to the Fuchsian group of signature $(g ; q)$. This defines our 2-orbifold bundle over the circle.

REMARK. In [17] this construction is carried out in detail for $M$ the complement of the figure-eight knot. In particular Jørgenson constructs the faithful discrete representation of the hyperbolic 3orbifold group into $\operatorname{PSL}(2, \mathbf{C})$.

3.3. The determination as to whether the orbifolds obtained by $(0, q)$-Dehn filling in the manner described above are arithmetic or not is simpler than the general case due in part to the following result implicit in [21].

LEMMA 2. Let $\Gamma$ be a non-elementary Kleinian group and $\Delta a$ non-trivial normal subgroup (possibly of infinite index). Then $k \Gamma=$ $k \Delta$ and $A \Gamma=A \Delta$.

Using this and the characterization theorems $A$ and $B$ of $\S 2$, we have; 
LeMmA 3. Let $Q=\mathbf{H}^{3} / \Gamma$ be a hyperbolic 2-orbifold bundle over the circle with fiber $S=\mathbf{H}^{3} / F$. Then if $F^{(2)}$ satisfies the three conditions of Theorem $B, \Gamma$ is arithmetic.

Proof. First of all, notice that as $F$ is normal in $\Gamma, F^{(2)}$ is normal in $\Gamma^{(2)}$. Thus $\Gamma^{(2)}$ is a subgroup of the normalizer of $F^{(2)}$ in $P S L(2, \mathbf{C})$. By assumption $F^{(2)}$ has integral traces, thus it follows from Proposition 2.8 of $[\mathbf{3}]$ that $\Gamma^{(2)}$, and hence $\Gamma$ has integral traces. Also by assumption $k F$ has one complex place and $A F$ is ramified at all real places. By Lemma 2 the same is true for $A \Gamma$. We therefore conclude that $\Gamma$ is arithmetic.

3.4. To complete $\S 3$ we discuss some further simplifications when the fiber is a torus with a single cone point with cone angle $2 \pi / q$.

Let $Q$ denote a hyperbolic 2-orbifold bundle over the circle with fiber a torus with a single cone point of cone angle $2 \pi / q$ arising from Dehn filling on the Mapping torus of $\phi$, a pseudo-Anosov selfhomeomorphism of a punctured torus. From the discussion in $\S 3.2$, a presentation for the orbifold fundamental group of $Q$ is,

$$
\Gamma_{Q}=\left\langle t, a, b \mid t a t^{-1}=\phi_{*}(a), t b t^{-1}=\phi_{*}(b),[a, b]^{q}=1\right\rangle .
$$

Let $\theta$ denote the faithful discrete representation of $\Gamma_{Q}$, and with the usual abuse of notation, let $x=\operatorname{tr}(\theta(a)), y=\operatorname{tr}(\theta(b))$ and $z=\operatorname{tr}(\theta(a b))$.

In $[\mathbf{2 6}]$, K. Takeuchi determines all arithmetic Fuchsian groups of signature $(1 ; q)$. The proof of Theorem 3.4 of $[\mathbf{2 6}]$ applies verbatim, to show, in conjunction with Lemma 2 ,

\section{LEMma 4. $k \Gamma_{Q}=\mathbf{Q}\left(x^{2}, y^{2}, z^{2}, x y z\right)$.}

To compute $x, y$, and $z$ we use the two equations determined by the action of $\phi$, i.e.,

$$
x=\operatorname{tr}\left(\theta\left(\phi_{*}(a)\right)\right) \text { and } y=\operatorname{tr}\left(\theta\left(\phi_{*}(b)\right)\right),
$$

together with the well-known trace identity for the trace of the commutator, which in the cases at hand has the form:

$$
x^{2}+y^{2}+z^{2}+x y z-2+2 \cos \pi / q=0 .
$$

Using multiple applications of resultants of these polynomials allows one to compute $x, y$ and $z$. This is effectively carried out using Mathematica. 
4. The Example. In this section we shall prove the following result.

THEOREM 1. There exists a pair of commensurable orientable non-Haken hyperbolic 3-manifolds which have a finite cover that fibers over the circle.

In section 4.1 we describe the non-Haken manifolds and in section 4.2 we describe how to construct a cover which is a bundle.

4.1. The first manifold is the 4 -fold cyclic branched cover of the figure eight knot. Denote this manifold by $M_{4}=\mathbf{H}^{3} / \Gamma_{4}$. That $M_{4}$ is non-Haken can be read off directly from the table in [7] where the boundary slopes of the 4-fold cyclic cover of the figure eight knot are listed (which of course is a 1-punctured torus bundle, so there are no closed essential surfaces). In particular the framing used is "the lift" of the standard meridian-longitude pair for the figure-eight knot. $M_{4}$ is known to be hyperbolic by [15]. Moreover $M_{4}$ is arithmetic. This was worked out for example in [13], where the invariant trace field was shown to be $\mathbf{Q}(\sqrt{-3})$ and the invariant quaternion algebra ramified at the $\mathbf{Q}(\sqrt{-3})$ primes above 2 and 3 .

The second manifold has the following surgery description. Let $X$ be an orientable 1-punctured torus bundle with monodromy $\phi$. Recall that $\phi$ can be interpreted as a hyperbolic element of $S L(2, \mathbf{Z})$. Using the generating pair $R=\left(\begin{array}{ll}1 & 1 \\ 0 & 1\end{array}\right)$ and $L=\left(\begin{array}{ll}1 & 0 \\ 1 & 1\end{array}\right)$ we can associate to any 1-punctured torus bundle the so-called RL-factorizationthis is non-unique but such things will not concern us here. Of interest to us is the 1-punctured torus bundle whose monodromy has the RL-factorization, $-R^{2} L$. Denote this manifold by $N$.

Now $N$ is an orientable hyperbolic 3-manifold of volume $2.66674478 \ldots$ (as can be checked using Snappea, [32], see also [1] for a discussion of this program ) and $H_{1}(N, \mathbf{Z}) \cong \mathbf{Z} \oplus \mathbf{Z}_{6}$. For the algebraic calculations that follow we find it convenient to use the description of $N$ as the census manifold m010, in the notation of $\mathrm{J}$. Weeks, [32], The closed manifold we require is obtained by $(-1,2)$ surgery on $N$.

LEMma 5. Let $M$ be the result of $(-1,2)$-Dehn filling on $N$. Then $M$ is a non-Haken arithmetic hyperbolic 3-manifold whose volume is 
that of the regular ideal tetrahedron in $\mathbf{H}^{3}$. The invariant quaternion algebra, which we will denote by $A$ is defined over $\mathbf{Q}(\sqrt{-3})$ and ramified at the primes above 2 and 3 in $\mathbf{Q}(\sqrt{-3})$.

Proof. We will make use of Mathematica in some of the calculations that follow. We include enough details so that the reader should find it is easy to reproduce the calculation using Mathematica.

That this manifold is hyperbolic follows from Snappea, where the volume coincides with that of regular ideal tetrahedron in $\mathbf{H}^{3}$ (really this is an approximation, but equality will follow from calculations below). To prove the statement concerning arithmeticity we shall make use of Theorems A and B of $\S 2$. Using Snappea, a presentation for $\pi_{1}(M)$ is given as follows:

$$
\left\langle a, b \mid b a^{2} b^{2} a^{-1} b^{-1} a^{-1} b=1, b^{-1} a b a^{3} b a b^{-1} a=1\right\rangle .
$$

We normalize a representation $\theta$ of $\pi_{1}(M)$ into $S L(2, \mathbf{C})$ such that:

$$
a=\left(\begin{array}{ll}
x & 1 \\
0 & x^{-1}
\end{array}\right) \text { and } b=\left(\begin{array}{ll}
y & 0 \\
r & y^{-1}
\end{array}\right),
$$

where $|x|$ and $|y|$ are greater than one. The idea now is simply to compute the irreducible polynomials of $x, y$ and $r$ corresponding to the hyperbolic structure, and then compute the invariant trace-field and algebra. This is efficiently done using multiple applications of resultants on Mathematica. We indicate how this argument goes.

Using each of the two relations we can eliminate $r$ using resultants, to give to equations in $x$ and $y$. For example, rewrite the first relation as a difference, $b a^{2} b^{2}-b^{-1} a b a=0$. One can eliminate $r$ using, for example, the $(1,1)$-entry and $(1,2)$-entry after clearing denominators to make these polynomials. Similarly write the second relation as a difference and eliminate $r$ using the $(1,1)$-entry and $(2,1)$-entry. In the first case, the resultant factors as a product of the following polynomials in $x$ and $y ;-1+y$ and $-x^{2}+y+$ $x^{2} y+x^{4} y-x^{2} y^{2}+y^{3}+x^{2} y^{3}+x^{4} y^{3}-x^{2} y^{4}$. As the image of the generators under the faithful discrete representation are hyperbolic, we can eliminate the first factor from our considerations. Denote the second factor by $p$. In the second case we get several trivial linear factors which can be eliminated by assumption that elements are 
hyperbolic, and one "large" polynomial factor in $x$ and $y$, namely $1+x^{4}+3 y^{2}+2 x^{2} y^{2}+3 x^{4} y^{2}+y^{4}+x^{4} y^{4}$. Denote this polynomial by $q$. We then proceed to eliminate $x$ and $y$ in turn using the polynomials $p$ and $q$ computed above. Eliminating $y$ gives (upon eliminating quadratic factors that correspond to the image of the generator being elliptic) $1+2 x^{2}+6 x^{4}+2 x^{6}+x^{8}$. This is the irreducible polynomial for $x$ that we require. A similar analysis for $y$ gives, $1-y+3 y^{2}-y^{3}+y^{4}$. Letting $z$ and $u$ denote $x+x^{-1}$ and $y+y^{-1}$ respectively we find that the hyperbolic structure corresponds to the solution;

$$
z^{4}-2 z^{2}+4=0 \text { and } u^{2}-u+1=0 .
$$

Thus $z^{2}=1 \pm \sqrt{-3}$ and $u=\frac{1 \pm \sqrt{-3}}{2}$.

To compute the invariant trace field, note that on clearing denominators the $(1,2)$-entry of $b a^{2} b^{2}-b^{-1} a b a$ is $-1+y+x^{2} y-$ $x^{2} y^{2}-x y r$. This must be zero. Rearranging this equation give $r+(x y+1 / x y)=x+1 / x$. The left hand side of this is simply the trace of $\theta(a b)$. Thus $\operatorname{tr}(\theta(a b))=\operatorname{tr}(\theta(a))$. Using [14] the invariant trace field is $\mathbf{Q}\left(z^{2}, u, \operatorname{tr}\left(a^{2} b^{2}\right)\right)$. Expanding $\operatorname{tr}\left(\theta\left(a^{2}\right) \theta\left(b^{2}\right)\right)$ via the trace identity $\operatorname{tr}(\theta(a b))+\operatorname{tr}\left(\theta\left(a^{-1} b\right)\right)=\operatorname{tr}(\theta(a)) \operatorname{tr}(\theta(b))$, and the fact that $\operatorname{tr}(\theta(a b))=\operatorname{tr}(\theta(a))$ from above, we obtain $\operatorname{tr}\left(\theta\left(a^{2}\right) \theta\left(b^{2}\right)\right)=$ $\operatorname{tr}(\theta(a))^{2}(\operatorname{tr}(\theta(b))-1)-\operatorname{tr}\left(\theta\left(b^{2}\right)\right)$, which from our calculations is an element of $\mathbf{Q}(\sqrt{-3})$. Thus the invariant trace-field is $\mathbf{Q}(\sqrt{-3})$.

Now $\operatorname{tr}(\theta(a))$, and $\operatorname{tr}(\theta(b))$ are algebraic integers, hence $\theta\left(\pi_{1}(M)\right)$ has integral traces. As there are no real embeddings to consider, we deduce from Theorems $\mathrm{A}$ and $\mathrm{B}$ that $\Gamma$ is arithmetic.

Let $\Gamma_{0}$ denote the faithful discrete representation of $\pi_{1}(M)$ computed above. To compute the algebra $A$, we can use volume estimates. First as $M$ is closed $A$ is a division algebra, see [31] for example. In the case at hand as $\mathbf{Q}(\sqrt{-3})$ has no real embeddings, $A$ must be ramified at some finite place of $\mathbf{Q}(\sqrt{-3})$. By Theorem $\mathrm{A}$, and our calculation above $\Gamma_{0}^{(2)}$ is derived from a quaternion algebra. It is easy to see from the presentation of $\pi_{1}(M)$ that the index of $\Gamma_{0}^{(2)}$ in $\Gamma_{0}$ is 2 . From the standard volume formula for the orbifolds $\mathbf{H}^{3} / P \rho\left(\mathcal{O}^{1}\right)$, see [5] we get an inequality using an approximation for the volume of $M$ from Snappea as the volume of the regular ideal tetrahedron to as many decimal places as we care to consider. This 
volume is 1.014 to three decimal places. Thus using this we get,

$$
2.03 \geq \frac{3^{3 / 2} \zeta_{\mathbf{Q}(\sqrt{-3})}(2) \prod_{\nu \in \operatorname{Ram}_{f}(A)}(N \nu-1)}{4 \pi^{2}}
$$

where $\operatorname{Ram}_{f}(A)$, the set of finite places of $\mathbf{Q}(\sqrt{-3})$ that ramify $A$, and $N \nu$ denotes the norm of the prime ideal corresponding to $\nu$. A calculation shows that the product above is at most 6 . As $M$ is closed, the algebra must be ramified at a finite set of places whose cardinality is even by the classification theory of quaternion algebras, see [31, Chapter 4]. It follows from the volume estimate that $A$ can only be ramified at the places above 2 and 3 .

By [5] or [19], Lemma 5 implies that $M$ and $M_{4}$ are commensurable. In fact we shall now show that $M_{4}$ is the unique double cover of $M$. It follows from this that $M$ is also non-Haken-this is also deduced form the results of [7] by computing the boundary slopes of the 1-punctured torus bundle $N$.

That $M$ has a unique double cover follows by computing $H_{1}(M, \mathbf{Z})$, which is $\mathbf{Z}_{3} \oplus \mathbf{Z}_{6}$. We collect some facts about the commensurability class of $M_{4}$ using the description of maximal arithmetic subgroups in a commensurability class due to Borel [5]. We shall only briefly recall some relevent points, and refer the reader to [5] for details.

Let $V$ denote the set of all places of $\mathbf{Q}(\sqrt{-3})$ and $\nu_{2}$ and $\nu_{3}$ the places above 2 and 3 respectively. According to [5], maximal arithmetic subgroups in the commensurablity class of $\Gamma_{4}$ are parametrized by pairs of disjoint subsets (finite and possibly empty) of $V \backslash\left\{\nu_{2}, \nu_{3}\right\}$ denoted by $S$ and $S^{\prime}$ respectively. Borel defines groups $\Gamma_{S, S^{\prime}}$, and every group commensurable with $\Gamma_{4}$ is conjugate to a subgroup of some such group. The minimal volume, say $\mu$ in the commensurability is achieved by groups $\Gamma_{\emptyset, S^{\prime}}$, and these form finitely many conjugacy classes. In fact, the group is unique in this case as the type number of $A$ is 1 , see [5], and [31]. Recall the type number of $A$, is the number of $A^{*}$-conjugacy classes of maximal orders, where $A^{*}$ denotes the invertible elements of $A$.

By definition $M_{4}$ is the 4 sheeted cyclic cover of the orbifold obtained by $(4,0)$-surgery on the figure-eight knot complement. Let $Q_{4}=\mathbf{H}^{3} / \Delta_{4}$ denote this orbifold. From Snappea we read off that $Q_{4}$ has volume approximately $v_{0} / 2$. 
Using the volume formula of Borel [5] we compute the smallest volume in the commensurability class to be $v_{0} / 8$. Let $\mathcal{O}$ be a maximal order of $A$. Using Borels description of maximal groups we see that there is a group $\Delta=\Gamma_{\emptyset, \emptyset}$ of co-volume $v_{0} / 8$ containing the group $P \rho\left(\mathcal{O}^{1}\right)$ of index 8. In particular $P \rho\left(\mathcal{O}^{1}\right)$ has volume $v_{0}$. Now $\Delta_{4}^{(2)}$ has index 2 in $\Delta_{4}$, as can be seen by using the presentation for $\Delta_{4}$ obtained by setting the fourth power of the meridian equal to 1 in the figure eight knot group. Consequently as $\Delta_{4}^{(2)}$ is derived from a quaternion algebra and has volume $v_{0}$ to as many decimal places as we care to compute, we have that $P \rho\left(\mathcal{O}^{1}\right)$ must coincide with $\Delta_{4}^{(2)}$ (up to conjugacy, using the fact that the type number is 1).

Now $\Gamma_{4}$ has index 2 in $\Delta_{4}^{(2)}$ and by conjugating if necessary, the fundamental group of the double cover of $M$ has index 2 in $\Delta_{4}^{(2)}$ as the double cover is also derived from a quaternion algebra (again making use of the type number of $A$ being 1 ). However, one can show that $\Delta_{4}^{(2)}$ has a unique subgroup of index 2 ; directly using a Reidemeister-Schreier rewriting procedure on $\Delta_{4}$ or use Cayley. Hence the claim is established.

4.2. We shall now consider the hyperbolic once punctured torus bundle with monodromy $\left(\begin{array}{ll}5 & 2 \\ 2 & 1\end{array}\right)$ and $q=2$. Let us fix notation for what follows by denoting by $Q$ the orbifold obtained by $(0,2)$-Dehn filling on this punctured torus bundle as described in Lemma 1 and denote by $T$ the fiber.

LEMma 6. $Q=\mathbf{H}^{3} / \Gamma_{1}$ is an arithmetic hyperbolic 3-orbifold commensurable with $M$ and $M_{4}$.

Proof. That $Q$ is hyperbolic can be checked directly using Snappea. Since the isomorphism class of a quaternion algebra determines the commensurability class of the arithmetic Kleinian groups, see [5] or [19], we are required to show that $\Gamma_{1}$ is arithmetic with the same invariant quaternion algebra as $\Gamma_{0}$. Let $F$ denote the orbifold fundamental group of $T$ as a subgroup of $\Gamma_{1}$.

To prove that $Q$ is arithmetic it suffices to show, in view of Lemmas 3 and 4 that (in the notation of $\S 3.4$ ) $x, y$ and $z$ are algebraic 
integers, that $\mathbf{Q}\left(x^{2}, y^{2}, z^{2}\right)=\mathbf{Q}(\sqrt{-3})$ - as the order of the commutator is 2 in this case, we see from the trace identity for the commutator that $x y z$ is already an element of the field $\mathbf{Q}\left(x^{2}, y^{2}, z^{2}\right)$-and the invariant quaternion algebra of $F$ is isomorphic to $A$.

Using the action of the monodromy as described in $\S 3.4$ we get the two polynomials

$$
\text { (1) } x=x\left(y^{2}-2\right)-z y+x \text {, (2) } y=x z-y \text {. }
$$

Together with the commutator identity of $\S 3.4$ with $q=2$, an elementary calculation using Mathematica yields the following polynomials that $x, y$ and $z$ satisfy. Our method was to take resultants of (1) with the expression for the commutator and (2) with the expression for the commutator, eliminating $z$ in both cases to get $x$ and $y$, then repeated eliminating $y$ to get $z$ :

$$
\begin{aligned}
& x^{4}\left(x^{4}-6 x^{2}+12\right)=0, \\
& y^{4}\left(y^{4}-6 y^{2}+12\right)=0, \\
& z^{4}\left(z^{2}-2\right)\left(z^{4}-4 z^{2}+16\right)=0 .
\end{aligned}
$$

The roots that correspond to the discrete faithful representation of $F$ as a subgroup of $\Gamma_{1}$ correspond to roots of the degree 4 factor of each of the above polynomials. The factor $\left(z^{2}-2\right)$ is redundant as there are no elements of order 4 in the orbifold group-the only singularities in $Q$ have cone angle $\pi$ along the core of the Dehn filling of the once-punctured torus bundle.

In particular notice that $x, y$ and $z$ are all algebraic integers. Furthermore, one sees that $x^{2}=y^{2}=3 \pm \sqrt{-3}$ and $z^{2}=2(1 \pm \sqrt{-3})$. By inspection the invariant trace-field is $\mathbf{Q}(\sqrt{-3})$.

To compute the quaternion algebra, we can use the description given in $\S 2.2$ where the non-commuting loxodromic elements we choose are the $\theta(a)$ and $\theta(b)$. Observe that since we know already that the algebra is defined over $\mathbf{Q}(\sqrt{-3})$ and the traces of $F^{(2)}$ are algebraic integers, the invariant quaternion algebra must be a division algebra of quaternions. Otherwise it would follow that the algebra is $M(2, \mathbf{Q}(\sqrt{-3}))$ and by the integral trace condition $F^{(2)}$ would be conjugate to a subgroup of $P S L\left(2, O_{3}\right)$ as the type number of this quaternion algebra is 1 (see [31, Chapter 1]). However $F^{(2)}$ is geometrically infinite and isomorphic to a cocompact Fuchsian 
group. This is impossible by the results of Bonahon and Thurston, cf. [4] and [27] which imply that (on dropping to a torsion free subgroup of finite index) a geometrically infinite surface group is virtually the fiber in a fibration over the circle. But torsion free subgroups of $\mathrm{PSL}\left(2, \mathrm{O}_{3}\right)$ are non-cocompact.

The calculations above show that the algebra is isomorphic to $\left(\frac{ \pm \sqrt{-3},-2}{\mathbf{Q}(\sqrt{-3})}\right)$. Hence the only $\mathbf{Q}(\sqrt{-3})$-primes that this algebra can be ramified at, are those above 2 and 3. By the previous paragraph, the quaternion algebra is ramified at some finite prime. It now follows from standard results in the theory of quaternion algebras, see [31, Chapter 3], that the algebra is isomorphic to $A$. This completes the proof of Lemma 6

The proof of Theorem 1 is now also completed.

4.3. In this section we compute the degree of the bundle cover of $M$ constructed above, and a topological description of how this bundle cover arises. We have had to make use of the group theoretic language Cayley to do this. All notation is that of $\S 4.1 \& 4.2$.

We begin with a preliminary remark on $Q$.

LEMMA 7. Q has a 2-fold quotient $Q^{\prime}$ which is a 2-orbifold bundle over the circle, the fiber being a sphere with 4 cone points, three of the cone angles being $\pi$ and the other $\pi / 2$.

Proof. The main observation in the proof is that a torus with a cone point of cone angle $\pi$ (regardless of the metric) has an involution whose quotient is a 2 -sphere with 4 cone points with cone angles in the statement of the lemma. Now such an involution extends to a 2-orbifold bundle with fiber a torus with a cone point of cone angle $\pi$.

Alternatively, a once-punctured torus bundle always has an orientation-preserving involution which quotients out the fiber to give a sphere with 3 cone points of appropriate cone angle and a puncture. The bundle required in the lemma is then a surgery on such an orbifold.

From Snappea we see that the volume of $Q$ is (approximately) $5 v_{0} / 2$, thus $Q^{\prime}$ has volume (approximately) $5 v_{0} / 4$. 
LEMMA 8. $M$ and $Q^{\prime}$ cover a common minimal orbifold.

Proof. As $\Gamma_{0}$ is 2-generator, there is an orientation-preserving involution on $M$ with one dimensional fixed point-set, see $[\mathbf{1 7}]$ and [27, Chapter 5]. Let $\tau$ denote this involution, and $\Lambda=\left\langle\Gamma_{0}, \tau\right\rangle$. The action of $\tau$ conjugates $a$ to $a^{-1}$ and $b$ to $b^{-1}$. With this, it is easy to see that $\Lambda$ can be presented as a group on three generators, $a$, $b$ and $\tau$, subject to the relations, $\tau^{2}=(\tau a)^{2}=(\tau b)^{2}=1$ together with those of $\Gamma_{0}$. An easy calculation shows the abelianization of $\Lambda$ is $\mathbf{Z}_{2} \oplus \mathbf{Z}_{2}$. Thus $\Lambda^{(2)}$ will be derived from a quaternion algebra of covolume $4 v_{0} / 2=2 v_{0}$. From the proof of Lemma 5 it follows that $\Lambda^{(2)}=\Gamma_{4}$. We claim $\Lambda$ is a subgroup of the maximal group $\Gamma_{\emptyset, \emptyset}$, recall $\S 4$.1. If $\Lambda$ contains the group $P \rho\left(\mathcal{O}^{1}\right)$ for some maximal order $\mathcal{O}$, then we are done, since the index is necessarily 2 , hence $P \rho\left(\mathcal{O}^{1}\right)$ is normal in $\Lambda$, and $\Lambda$ is therefore a subgroup of the normalizer of $P \rho\left(\mathcal{O}^{1}\right)$. This group coincides (up to conjugacy in the case at hand-recall the type number of $A$ is 1 ) with $\Gamma_{\emptyset, \emptyset}$. To see this we simply note that an alternative description of $\Gamma_{\emptyset, \emptyset}$ is $P \rho(\operatorname{Norm}(\mathcal{O}))$, where $\operatorname{Norm}(\mathcal{O})=\left\{x \in A: x \mathcal{O} x^{-1}=\mathcal{O}\right\}$. As norm is preserved by conjugation, the normalizer in $\operatorname{PSL}(2, \mathbf{C})$ of $P \rho\left(\mathcal{O}^{1}\right)$ coincides with $\operatorname{P} \rho(\operatorname{Norm}(\mathcal{O}))$.

If $\Lambda$ does not contain $P \rho\left(\mathcal{O}^{1}\right)$, we still have that $\Lambda$ and $P \rho\left(\mathcal{O}^{1}\right)$ are subgroups of the normalizer in $P S L(2, \mathbf{C})$ of $\Gamma_{4}$, which by assumption will be a proper supergroup of $\Lambda$. Such a group has covolume at most $v_{0} / 4$.

An analysis of primes of small norm in $\mathbf{Q}(\sqrt{-3})$ together with Borels description of maximal groups in this commensurability class can be used to justify the claim. From the volume formula the only ones to worry about are, $\Gamma_{\nu_{7}, S^{\prime}}$ and $\Gamma_{\nu_{7}^{\prime}, S^{\prime}}$ where $\nu_{7}$ and $\nu_{7}^{\prime}$ denote the pair of places dividing 7 in $\mathbf{Q}(\sqrt{-3})$. However Borel's volume formula rule these out. Thus the only possible supergroup of the normalizer of $\Gamma_{4}$ can be $\Gamma_{\emptyset, \emptyset}$. The claim is established.

Let $Q^{\prime}=\mathbf{H}^{3} / \Gamma_{2}$. We now show that $\Gamma_{2} \subset \Gamma_{\emptyset, \emptyset}$.

As above, if $\nu_{7}$ and $\nu_{7}^{\prime}$ denote the pair of places dividing 7 in $\mathbf{Q}(\sqrt{-3})$, Borel's volume formula show that $Q^{\prime}$ cannot cover an orbifold obtained by the quotient of $\mathbf{H}^{3}$ by either of the groups $\Gamma_{\nu_{7}, S^{\prime}}$ and $\Gamma_{\nu_{7}^{\prime}, S^{\prime}}$. Hence $\Gamma_{2} \subset \Gamma_{\emptyset, \emptyset}$ as required. 
By volume considerations, we see that $\Gamma_{0}$ is a subgroup of $\Gamma_{\emptyset, \emptyset}$ of index 8, and $\Gamma_{2}$ is a subgroup of $\Gamma_{\emptyset, \emptyset}$ of index 10. Consequently, $\Gamma_{0} \cap \Gamma_{2}$ has index at most 10 in $\Gamma_{0}$. As $\Gamma_{0} \cap \Gamma_{2}$ is torsion-free, it must have index 2, 4, 6 or 8 in $\Gamma_{2}$. The cases where $\Gamma_{0} \cap \Gamma_{1}$ has index 2, 4 or 6 in $\Gamma_{2}$ are impossible for Euler characteristic reasons and the fact that $\Gamma_{0} \cap \Gamma_{2}$ is torsion-free. It follows that the index is 8 and the fiber is genus 2 . With this we have.

LEMma 9. The cover of $M$ corresponding to $\Gamma_{0} \cap \Gamma_{2}$ has degree 10, and is a genus 2 surface bundle over the circle. The first homology of this bundle is $\mathbf{Z} \oplus \mathbf{Z}_{6} \oplus \mathbf{Z}_{6}$.

Proof. All that remains to check is that the homology is $\mathbf{Z} \oplus \mathbf{Z}_{6} \oplus$ $\mathbf{Z}_{6}$. However it can be shown using Cayley that $\Gamma_{0}$ has a unique subgroup of index 10 with infinite abelianization.

One can get a more refined picture of how this bundle cover occurs. Let $\widetilde{M}=\mathbf{H}^{3} / G$ denote the bundle cover given by Lemma 9 . Using Cayley one sees that $\Gamma_{0}$ has a unique subgroup of index 5 up to conjugacy. This group has abelianization $\mathbf{Z}_{2} \oplus \mathbf{Z}_{6} \oplus \mathbf{Z}_{6}$. Furthermore, this group contains $G$ as a subgroup of index 2 . As $H_{1}(\widetilde{M} ; \mathbf{Z})$ has rank 1 , and hence by duality $H_{2}(\widetilde{M} ; \mathbf{Z})$ has rank 1 it follows from $\left[\mathbf{9}\right.$, Corollary 3.3] that the manifold obtained by the quotient of $\mathbf{H}^{3}$ by the subgroup of index 5 is Haken. Indeed the following argument proves it is a union of two twisted I-bundles.

LEMMA 10. Let $X$ be a closed irreducible orientable 3-manifold such that $H_{1}(X, \mathbf{Z})$ is finite, and $Y$ is a double cover of $X$ which fibers over the circle with first betti number equal one. Then $X$ is the union of two twisted I-bundles.

Proof. We shall show that the hypothesis imply that there is a map from $\pi_{1}(X)$ to $\mathbf{Z}_{2} * \mathbf{Z}_{2}$ induced from a map of $\pi_{1}(Y)$ onto $\mathbf{Z}$ with finitely generated kernel. By [10, Chapter 11] $X$ is a union of two twisted I-bundles.

The double cover $Y \longrightarrow X$ corresponds to a free $\mathbf{Z}_{2}$ action on $Y$. Denote the involution by $\tau$. The involution acts by conjugation on $\pi_{1}(Y)$, which descends to an involution on the $\mathbf{Z}$-factor by factoring through $H_{1}(Y, \mathbf{Z})$. We continue to denote the involution by $\tau$. 
Let $y$ be an element of $\pi_{1}(Y)$ whose image $\bar{y}$ generates the $\mathbf{Z}$ factor in homology. $\tau$ acts on this factor by sending $y$ to $\bar{y}$ or $-\bar{y}$. But then there is an induced map from $\pi_{1}(X)$ to a group $K$ which coincides with the extension of $\mathbf{Z}$ determined by the action of $\tau$. The possible extensions are either $\mathbf{Z} \oplus \mathbf{Z}_{2}$ in the case $y$ maps to $\bar{y}$, or $\mathbf{Z}_{2} * \mathbf{Z}_{2}$ in the case $y$ maps to $-\bar{y}$. As $H_{1}(X, \mathbf{Z})$ is finite, we have the required map to $\mathbf{Z}_{2} * \mathbf{Z}_{2}$. By the assumption that $H_{1}(Y, \mathbf{Z})$ has rank 1, the involution maps a fiber to something isotopic to it. It follows that the kernel of the induced map to $\mathbf{Z}_{2} * \mathbf{Z}_{2}$ is finitely generated.

We can also describe the cover of $M_{4}$. Arguing as above we see the appropriate cover of $M_{4}$ has degree 10. Again, using Cayley there is precisely one conjugacy class of subgroups of $\Gamma_{4}$ of index 10 with infinite abelianization, namely $\mathbf{Z} \oplus \mathbf{Z}_{12} \oplus \mathbf{Z}_{24}$. This bundle is a degree two cover of $\widetilde{M}$. As both have rank 1 first homology the bundle cover of $M_{4}$ has also a fiber of genus 2 . The cover is obtained by unwrapping in the circle direction, so the fiber lifts.

Furthermore, as $M$ and $M_{4}$ are arithmetic with covers with positive first betti number, it follows from, for example [6], that there are finite covers of $M$ and $M_{4}$ where the betti number can be made arbitrarily large. Thus by the properties of the Thurston Norm [30, Corollary to Theorem 3.3], there is a cover of $M$ and $M_{4}$ containing an embedded quasi-Fuchsian surface. In addition by the results of [19] and [22] the quasi-Fuchsian surface cannot be totally geodesic. As a corollary of these remarks we see that,

COROLlaRY. $M$ and $M_{4}$ contain immersions of both geometrically infinite surfaces and quasi-Fuchsian, non totally geodesic surfaces.

5. Final Remarks. We conclude with a couple of remarks.

5.1. Other cyclic branched covers of the figure eight knot are also finitely covered by a surface bundle, although not in an interesting manner! Denote by $M_{n}$ the $n$-fold cyclic branched cover of the figure eight knot.

TheOREM 2. For all positive integers $k$, the manifolds $M_{3 k}$ are 
the union of two twisted I-bundles over a non-orientable surface and hence double covered by a fiber bundle over the circle. For $k>1$, the manifolds are hyperbolic.

Sketch Proof. Firstly the manifold $M_{3}$ is a Euclidean manifold which can be described as the union of two twisted I-bundles over the Klein Bottle. This manifold is double covered by a manifold with first betti number equal one that fibers over the circle with fiber the 2-torus. Hyperbolicity for those $M_{3 k}$ with $k>1$ follows from $[27]$.

The branch cover $M_{3 k} \longrightarrow S^{3}$ branched over the figure eight knot factors through the 3 -fold branched cover. It can be shown that the preimage of the figure eight knot in $M_{3}$ can be made transverse to a torus isotopic to one which lifts in the double cover obtained by unwrapping the I-bundle pieces. One can then pull this "union of two twisted I-bundle structure" through the $3 k$-fold branched covers using the fact in the double cover of $M_{3}$ the preimage of the figure eight knot will be transverse to the fibration. It is well-known that such fibrations can be pulled-back through such branched covers

5.2. Lemma 10 should be compared with the constructions of [8]. The content of Lemma 10 is that Gabai's construction does not work if the bundle upstairs has only rank 1 first homology. For the argument in Lemma 10 shows either we have a union of two twisted I-bundles or the manifold downstairs is also fibered; the case of the union of two twisted I-bundles was dealt with, the other case is when the $\mathbf{Z}_{2}$-extension is $\mathbf{Z} \oplus \mathbf{Z}_{2}$, and the map to $\mathbf{Z}$ induced on the quotient is also induced by a fibering.

5.3. Although many classes of hyperbolic 3-manifolds have been shown to have finite covers with positive first betti number, see for example [2], [11], [12], and [18], little is known, even in the cases where positive first betti number can be shown in finite covers, in regard to having finite covers which are fibered over the circle. Expanding on this, if $M=\mathbf{H}^{3} / \Gamma$ is a closed hyperbolic 3-manifold that fibers over the circle and $S$ is a fiber, then the induced representation of $\pi_{1}(S)$ as a subgroup of $\Gamma$ is geometrically infinite since the limit set is the entire sphere-at-infinity. Unfortunately there seems little at present that allows one to distinguish algebraically or group theoretically whether a subgroup of a Kleinian group of finite co-volume 
which is isomorphic to a surface group is geometrically infinite or geometrically finite without a priori knowing additional information about this surface group. Recent work along these lines has been done by T. Soma in [23] and [24].

Acknowledgements. This work was supported in part by the S.E.R.C. (U.K.) and N.S.F. Grants DMS-8505550 administered through M.S.R.I. and DMS-9108050. The work was completed whilst the author was visiting The University of Melbourne. The author wishes to thank M.S.R.I. and The University of Melbourne for their hospitality. He also thanks the members of The Geometry and Topology Group at The University of Melbourne and Darren Long for many useful conversations on subjects related to the contents of this paper.

\section{REFERENCES}

[1] C. C. Adams, SNAPPEA, the Weeks hyperbolic three-manifolds program, Notices Amer. Math. Soc., 37 (1990), 273-275.

[2] M. D. Baker, Covers of Dehn fillings on once-punctured tors bundles, Proc. Amer. Math. Soc., 105 (1989), 747-754.

[3] H. Bass, Groups of integral representation type, Pacific J. Math., 86 (1980), 15-51.

[4] F. Bonahon, Bouts des variétiés hyperbolique de dimension 3, Annals of Math., 124 (1986), 71-158.

[5] A. Borel, Commensurability classes and volumes of hyperbolic 3-manifolds, Ann. Scuola Norm. Sup. Pisa, 8 (1981), 1-33.

[6] A. Borel, Cohomologie de sous-groupes discrets et représentations de groupes semi-simples, Astérisque, 32-33 (1976), 73-112.

[7] M. Culler, W. Jaco and H. Rubinstein, Incompressible surfaces in once punctured torus bundles, Proc. London Math. Soc., 45 (1982), 385-419.

[8] D. Gabai, On 3-manifolds finitely covered by suface bundles. In Lowdimensional Topology and Kleinian Groups, Ed. D. B. A. Epstein, London Math. Soc. Lecture Note Series, 112 (1986), 145-155.

[9] J. Hass, Surfaces minimizing area in their homology class and group actions on 3-manifolds, Math. Zeit., 199 (1988), 501-509.

[10] J. Hempel, 3-Manifolds, Annals of Math. Study 86, Princeton University Press.

[11] J. Hempel, Orientation reversing involutions and the first betti number for finite coverings of 3-manifolds, Invent. Math., 67 (1982), 133-142. 
[12] J. Hempel, Coverings of Dehn fillings of surface bundles I, \& II, Topology and its Appl., 24 (1986), 157-170, ibid. 26 (1987), 163-173.

[13] H. M. Hilden, M. T. Lozano and J-M. Montesinos, Arithmeticity of figure eight knot orbifolds, in Topology'90, Proceedings of the Research Semester in Low-Dimensional Topology at Ohio State University, De Gruyter Verlag, 1992.

[14] H. M. Hilden, M. T. Lozano and J-M. Montesinos, A characterization of arithmetic subgroups of $S L(2, \mathbf{R})$ and $S L(2, \mathbf{C})$, preprint.

[15] C. D. Hodgson, Ph. D Thesis, Princeton University, 1986.

[16] T. Jørgenson, On pairs of once punctured tori, preprint.

[17] T. Jørgenson, Compact 3-manifolds of constant negative curvature fibering over the circle, Annals of Math., 106 (1977), 61-72.

[18] D. D. Long, Immersions and embeddings of totally geodesic surfaces, Bull. London Math. Soc., 19 (1987), 481-484.

[19] C. Maclachlan and A. W. Reid, Commensurability classes of arithmetic Kleinian groups and their Fuchsian subgroups, Math. Proc. Camb. Phil. Soc., 102 (1987), 251-257.

[20] W. D. Neumann and A. W. Reid, Arithmetic of hyperbolic 3-manifolds, in Topology'90, Proceedings of the Research Semester in Low-Dimensional Topology at Ohio State University, De Gruyter Verlag, 1992.

[21] W. D. Neumann and A. W. Reid, Amalgamation and the invariant-trace field of a Kleinian group, Math. Proc. Camb. Phil. Soc., 109 (1991), 509-515.

[22] A. W. Reid, Ph. D Thesis, University of Aberdeen, 1987.

[23] T. Soma, Virtual fibers in hyperbolic 3-manifolds, Topology and its Appl., 41 (1991), 179-192.

[24] T. Soma, Virtual fiber groups in 3-manifold groups, J. London Math. Soc., 43 (2) (1991), 337-354.

[25] J. Stallings, On fibering certain 3-manifolds, in Toplogy of 3-Manifolds Ed. M. Fort Jr., 95-100, Prentice-Hall 1962.

[26] K. Takeuchi, Arithmetic Fuchsian groups of signature $(1 ; e)$, J. Math. Soc. Japan, 35 (1981), 381-407.

[27] W. P. Thurston, The Geometry and Topology of 3-Manifolds, Mimeographed lecture notes, Princeton University, 1977.

[28] W. P. Thurston, Three-dimensional manifolds, Kleinian groups and hyperbolic geometry, Bull. Amer. Math. Soc., 6 (1982), 357-381.

[29] W. P. Thurston, Hyperbolic structures on 3-manifolds II: surface groups and 3-manifolds that fiber of the circle, to appear Annals of Math.

[30] W. P. Thurston, A norm for the homology of 3-manifolds, Memoirs of the Amer. Math. Soc., 339 (1986), 99-130. 
[31] M-F. Vignéras, Arithmétique des Algèbres de Quaternions. L. N. M 800, Springer-Verlag 1980.

[32] J. Weeks, Snappea, Version 5/18/92, A program for the Macintosh to compute hyperbolic structures on 3-manifolds, Available upon request from J. Weeks.

Received August 18, 1992 and in revised form September 8, 1992.

Mathematical Sciences Research Institute 1000 Centennial Drive

BERKELEY, CA 94720

Current address:

DPMMS

UNIVERSITY OF CAMBRIDGE

CAMBRIDGe CB2 1SB

ENGLAND

E-mail address: areid@pmms.cam.ac.uk 


\title{
PACIFIC JOURNAL OF MATHEMATICS
}

Founded by

\author{
E. F. BeCKenBACH (1906-1982) F. Wolf (1904-1989)
}

EDITORS

Sun-Yung A. Chang

(Managing Editor)

University of California

Los Angeles, CA 90024-1555

pacific@math.ucla.edu

F. Michael Christ

University of California

Los Angeles, CA 90024-1555

christ@math.ucla.edu

ThOMAS ENRIGHT

University of California

San Diego, La Jolla, CA 92093

tenright@ucsd.edu
Nicholas ERCOLANI

University of Arizona

Tucson, AZ 85721

ercolani@math.arizona.edu

R. FINN

Stanford University

Stanford, CA 94305

finn@gauss.stanford.edu

VAUGhan F. R. Jones

University of California

Berkeley, CA 94720

vfr@math.berkeley.edu

SteVEn KeRcKHofF

Stanford University

Stanford, CA 94305

spk@gauss.stanford.edu
MARTIN SCHARLEMANN

University of California

Santa Barbara, CA 93106

mgscharl@math.ucsb.edu

\section{GANG TIAN}

Courant Institute

New York University

New York, NY 10012-1110

tiang@taotao.cims.nyu.edu

V. S. VARADARAJAN

University of California

Los Angeles, CA 90024-1555

vsv@math.ucla.edu

\section{SUPPORTING INSTITUTIONS}

UNIVERSITY OF ARIZONA

UNIVERSITY OF BRITISH COLUMBIA

CALIFORNIA INSTITUTE OF TECHNOLOGY

UNIVERSITY OF CALIFORNIA

UNIVERSITY OF MONTANA

UNIVERSITY OF NEVADA, RENO

NEW MEXICO STATE UNIVERSITY

OREGON STATE UNIVERSITY

\author{
UNIVERSITY OF OREGON \\ UNIVERSITY OF SOUTHERN CALIFORNIA \\ STANFORD UNIVERSITY \\ UNIVERSITY OF HAWAII \\ UNIVERSITY OF UTAH \\ WASHINGTON STATE UNIVERSITY \\ UNIVERSITY OF WASHINGTON
}

The Supporting Institutions listed above contribute to the cost of publication of this Journal, but they are not owners or publishers and have no responsibility for its content or policies.

Mathematical papers intended for publication in the Pacific Journal of Mathematics should be in typed form or offset-reproduced (not dittoed), double spaced with large margins. Please do not use built up fractions in the text of the manuscript. However, you may use them in the displayed equations. Underline Greek letters in red, German in green, and script in blue. The first paragraph must be capable of being used separately as a synopsis of the entire paper. In particular it should contain no bibliographic references. Please propose a heading for the odd numbered pages of less than 35 characters. Manuscripts, in triplicate, may be sent to any one of the editors. Please classify according to the 1991 Mathematics Subject Classification scheme which can be found in the December index volumes of Mathematical Reviews. Supply name and address of the author to whom proofs should be sent. All other communications should be addressed to the managing editor, or Julie Honig, University of California, Los Angeles, California 90024-1555.

There are page-charges associated with articles appearing in the Pacific Journal of Mathematics. These charges are expected to be paid by the author's University, Government Agency or Company. If the author or authors do not have access to such Institutional support these charges are waived. Single authors will receive 75 free reprints; joint authors will receive a total of 100 free reprints. Additional copies may be obtained at cost in multiples of 50 .

The Pacific Journal of Mathematics (ISSN 0030-8730) is published monthly except for July and August. Regular subscription rate: $\$ 215.00$ a year (10 issues). Special rate: $\$ 108.00$ a year to individual members of supporting institutions.

Subscriptions, orders for numbers issued in the last three calendar years, and changes of address should be sent to Pacific Journal of Mathematics, P.O. Box 4163, Berkeley, CA 94704-0163, U.S.A. Old back numbers obtainable from Kraus Periodicals Co., Route 100, Millwood, NY 10546.

The Pacific Journal of Mathematics at University of California, c/o Department of Mathematics, 981 Evans Hall, Berkeley, CA 94720 (ISSN 0030-8730) is published monthly except for July and August. Second-class postage paid at Berkeley, CA 94704, and additional mailing offices. POSTMASTER: send address changes to Pacific Journal of Mathematics, P.O. Box 4163, Berkeley, CA 94704-0163.

PUBLISHED BY PACIFIC JOURNAL OF MATHEMATICS at University of California,

Berkeley, CA 94720, A NON-PROFIT CORPORATION

This publication was typeset using $\mathcal{A} \mathcal{M} \mathcal{S}$ - IATEX,

the American Mathematical Society's TEX macro system.

Copyright (C)1994 by Pacific Journal of Mathematics 


\section{PACIFIC JOURNAL OF MATHEMATICS}

Volume $167 \quad$ No. $1 \quad$ January 1995

Local reproducing kernels on wedge-like domains with type 2 edges

Al Boggess and AlexANDER NAGEL

Discriminants of involutions on Henselian division algebras

MaUrice Chacron, H. DHERTE, JEAN-Pierre Tignol,

ADRIAN R. WADSWORTH and V. I. YANCHEVSKIĬ

Essential tori obtained by surgery on a knot

MARIO EUdAVE-MuÑOZ

Non-compact totally peripheral 3-manifolds

LUKE HARRIS and PETER SCOTT

Some representations of TAF algebras

JOHN LINDSAY ORR and JUSTIN PETERS

A non-Haken hyperbolic 3-manifold covered by a surface bundle

ALAN W. REID

The nonhomogeneous minimal surface equation involving a measure 